\title{
Nonlinear Diffusion Combined with Brownian Motions for Image Denoising
}

\author{
Mingliang Guo ${ }^{1} \quad$ Peng Liu $^{2}$ \\ ${ }^{1}$ Electronics and Information College, Heilongjiang Institute of Science and Technology, \\ Harbin 150027, China \\ ${ }^{2}$ College of Computer Science and Technology, Harbin Institute of Technology, Harbin
} 150001, China

\begin{abstract}
:
We introduce Brownian motion into the nonlinear diffusion process for image denoising in this paper. Brownian motion in our work stands for the variation of gray value of a pixel in the form of "random walking" which analogs the irregular and unceasing movement of a small particle. A reflection wall is set for each pixel to force its Brownian motion to act as an inverse process of the diffusion process. Moreover, the absorption walls are also set to limit the range of the distance of Brownian motion in order to overcome the inherent unstability of the inverse process. The parameters of Brownian motion and the location of the reflection and absorption walls are estimated adaptively according to the local feature of the neighborhood of the corresponding pixel. The method of the combination of the nonlinear diffusion and Brownian motion is called as ND-BM method. The experimental results shows that it is one of effective method for image denoising.
\end{abstract}

Keywords:Image denoising; PDEs; nonlinear diffusion; Brownian motion

*This work was supported by National Natural

Science Foundation of China (60702032),

Natural Scientific Research Innovation

Foundation HIT (HIT.NSRIF.2008.63)

\section{Introduction}

Many applications are depended on images quality. However, those observed images are not always good enough because of various reasons. Some noise is ineluctably introduced into imaging equipments and transmission channels. Image denoising is historically one of the oldest and important concerns in image processing. It is still a challenging and open research field.

There are many methods to tackle the image denoising problem. Diffusion filter is one of successful methods and can be formulated in terms of Partial Differential Equations(PDEs). The most investigated diffusion filter in image processing is the heat propagation equation[1]. Image denoising by diffusion filter is equivalent to carry out a Gaussian linear filter, and the denoisy image would be the mean value of the noisy image as the diffusion time tends to infinity. Therefore, it has some drawbacks: It is too smoothing and edges are lost. This problem was overcome in some extend by nonlinear diffusion filter proposed by

Perona and Malik(P-M)[2]. The basic ideal behind P-M method is that inside the regions where the magnitude of the gradient is weak, the diffusion is executed in faster speed than those regions where the magnitude of the gradient is strong. Some limitation and

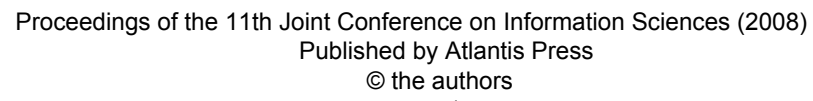


drawbacks of P-M method have been mentioned in [3]. In fact, P-M method also blurs the edges in some extend, although its diffusion speed is slow in edges regions. A forward and backward(FAB) diffusion processes for image denoising was proposed by Giboa[4]. FAB method can remove noise while preserve the edges at best, but the parameters of both forward and backward diffusion processes must be selected carefully in order to guarantee the convergence of the iteration process.

Our work is to extend the nonlinear diffusion methods by combining it with the small particle physics theory-Brownian motion. The diffusion can be regarded as heat propagation process; its goal is that all particles are homomorphic. On the contrary, Brownian motion is regarded as confusion process, referred to the irregular and unceasing movement. Such natural property of Brownian motion is utilized to preserve the edges more effectively in this proposed method for image denoising. Furthermore, the reflection and absorption walls are adaptively set for sampled points in image so that the combination of nonlinear diffusion and Brownian motion, called ND-BM is convergent. The experimental results show that the ND-BM is one of effective method for image denoising.

\section{Nonlinear diffusion for Image Denoising}

\subsection{The mathematical model of image denoising}

A commonly used model for image denoising is described as follows: An original image $u: \Omega \subset R^{2} \rightarrow R$ represents a real scene or called an original image, and let $u_{0}$ denote the noisy image or called the observed image. The image degradation model is written as:

$$
u_{0}=u+\eta
$$

where $\eta$ stands for a white additive Gaussian noise. Image denoising is indeed signal decomposition problem. This is an ill-posed problem and its solution is only an approximation of original image $u$.

\subsection{Image denoising by nonlinear diffusion}

Nonlinear diffusion is to obtain a family $u(x, t)$ of filtered versions of the noisy image $u_{0}=u(x, 0)$ as the solution of the nonlinear diffusion process under Neumann boundary conditions[2]:

$$
\begin{cases}\frac{\partial u(x, t)}{\partial t}=\operatorname{div}\left(c\left(|\nabla u|^{2}\right) \nabla u\right) & \text { in } \boldsymbol{\Omega} \times(0, T) \\ \frac{\partial u(x, t)}{\partial N}=0 & \partial \boldsymbol{\Omega} \times(0, T) \\ u(x, 0)=u_{0}(x) & \text { in } \boldsymbol{\Omega}\end{cases}
$$

where $x=\left(x_{1}, x_{2}\right)$ denotes the space coordinate, and $c\left(|\nabla u|^{2}\right):[0,+\infty) \rightarrow[0,+\infty)$ is a decreasing function of square of the gradient, called diffusion function. If $c\left(|\nabla u|^{2}\right) \equiv 1$ then Eq.(2) describes a linear diffusion process. The tangent and parallel directions to the gradients are denoted by $T$-direction and $N$-direction, respectively. Then $u_{T T}$ and $u_{N N}$ are the second derivation of $u$ in $T$-direction and $N$-direction[5]:

$$
\begin{aligned}
& u_{T T}=\frac{1}{|\nabla u|^{2}}\left(u_{x_{1}}^{2} u_{x_{2} x_{2}}+u_{x_{2}}^{2} u_{x_{1} x_{1}}-2 u_{x_{1}} u_{x_{2}} u_{x_{1} x_{2}}\right) \\
& u_{N N}=\frac{1}{|\nabla u|^{2}}\left(u_{x_{1}}^{2} u_{x_{1} x_{1}}+u_{x_{2} 2}^{2} u_{x_{2} x_{2}}+2 u_{x_{1}} u_{x_{2}} u_{x_{1} x_{2}}\right)
\end{aligned}
$$

where the subscripts denote partial derivatives. The first equation in Eq.(2) can be written as follows by using $u_{T T}$ and $u_{N N}$ : 
$\frac{\partial u(x, t)}{\partial t}=c\left(|\nabla u|^{2}\right) u_{T T}+b\left(|\nabla u|^{2}\right) u_{N N}$

where $b\left(|\nabla u|^{2}\right)=c\left(|\nabla u|^{2}\right)+2|\nabla u|^{2} c^{\prime}\left(|\nabla u|^{2}\right)$. One of classical diffusion functions is $c\left(|\nabla u|^{2}\right)=1 / \sqrt{1+|\nabla u|^{2}}$. In the next section, we will see that the second derivations $u_{T T}$ and $u_{N N}$ are used to estimate the parameters of Brownian motion and control the range of the variation of gray value of each pixel.

\section{Brownian Motion for Edge Preservation}

Brownian motion is a sophisticated random process, which was discovered by R.Brown in 1827. Brownian motion is referred as the irregular and unceasing movement of small particles when they suspend in a fluid medium. Many theories and applications related to it have been developed and played major roles in the real world. In this paper, we introduce Brownian motion into image deoising, which combines with nonlinear diffusion so as to remove noise while preserve the edges more effectively.

Nonlinear diffusion has capability to preserve the edges in image, because it adopts the different diffusion speed according to the local feature of image: inside the region where the magnitude of the gradient of $u$ is small, the diffusion is executed at high speed since the diffusion function $c\left(|\nabla u|^{2}\right)$ takes larger value; on the contrary, near edges region where the magnitude of the gradient of $u$ is large, the diffusion is executed at slow speed, since $c\left(|\nabla u|^{2}\right)$ has smaller value. Although taking different speed for different regions, the edges are blurred indeed to some extend, while removing noise.

\subsection{The active degree of pixel}

We regard a sampled point(pixel) in image as a small particle. Brownian motion causes it random movement, this process happens at the same time of diffusion process. We view the displacement caused by Brownian motion as the variation of gray value of pixel. Based on the properties of Brownian motion, the displacement is independent to each pixel and it is affected by the temperature of this pixel. It is clear that a pixel(particle) which has higher temperature has more active degree, and more active degree may cause the longer displacement. We use the norm of the second derivation vector $\left[u_{T T}, u_{N N}\right]^{T}$ to stand for the active degree of pixel $u(x, t)$ at the diffusion time $t$ :

$$
\alpha(x, t)=\sqrt{u_{T T}^{2}(x, t)+u_{N N}^{2}(x, t)}
$$

The pixel that is on the edge has more active degree than one that is in the smoothness regions.

\subsection{The displacement of Brownian motion}

At time $t$, the displacement of a particle subjects to normal distribution with mean value zero and variance $\sigma^{2}$. In other word, let $\nabla g_{B}(x, t)$ denotes the variation of gray value of pixel $u(x, t)$ at time $t$, which is caused by Brownian motion, then $\nabla g_{B}(x, t) \sim N\left(0, \sigma^{2}\right)$. The parameter $\sigma=\sigma(x, t)>0$ controls the probability distribution of the displacement. It is clear that $\sigma$ should increase as active degree $\alpha=\alpha(x, t)$ also increases. So it expression can be written as:

$$
\sigma(x, t)=w \times \alpha(x, t)
$$

where $w$ is a weight value. We normalizes the active degree of all pixels as follows:

$$
\breve{\alpha}(x, t)=\frac{\alpha(x, t)-\min \{\alpha(x, t), x \in \Omega\}}{\max \{\alpha(x, t), x \in \Omega\}-\min \{\alpha(x, t), x \in \Omega\}}
$$

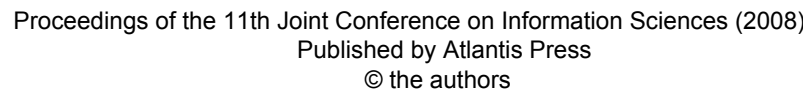


$\breve{\alpha}(x, t) \in[0,1]$. And the mean value of $\breve{\alpha}(x, t), x \in \Omega$ is

$$
\bar{\alpha}(t)=\frac{1}{|\Omega|} \sum_{x \in \Omega} \bar{\alpha}(x, t)
$$

where $|\Omega|$ denotes the number of the total pixels in an image $u$. Then an adaptive weight value $w=w(x, t)$ is computed by:

$w(x, t)=\frac{1}{2}\left\{1+\operatorname{sign}(\breve{\alpha}(x, t)-\overline{\bar{\alpha}}(t)) \times(\breve{\alpha}(x, t)-\overline{\bar{\alpha}}(t))^{(k-1)}\right\}$

where $k \in[1,2]$. The curve of $w=w(x, t)$ with a constant time $t$ and $\overline{\bar{\alpha}}(t)=0.25$ is drawn in Fig.1. In our study, we let $k=1.1$.

From the Fig.1 and Eq.(7), we can find that the weight coefficient $w$ adjusts the effect of active degree $\alpha(x, t)$ to the standard deviation $\sigma$ of Brownian motion at location $x$ and time $t$. As increasing of $w$ and $\alpha(x, t)$, $\sigma$ gets large value. It means that the pixel having more active degree has more possibility to make longer Brownian motion displacement than the one that has smaller active degree. For the pixel $u(x, t)$, its Brownian motion displacement $\nabla g_{B}(x, t)$ is a normal distribution random number:

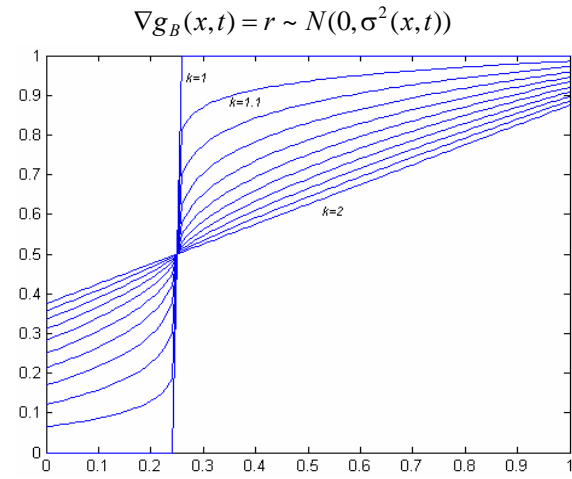

Fig1. The curves family of weight coefficients

\subsection{Reflection walls and absorption} walls

Assuming that $\hat{x}$ is an edge pixel location of image $u$ at diffusion time $t$. we will say that $\hat{x}$ is blurred in $N$-direction if $u_{N N}(x, t)$ decreases as $t$ increases, i.e. if $\frac{\partial u_{N N}}{\partial t}(x, t)<0$, and noise is amplified in $T$-direction if $u_{T T}(x, t)$ increases as $t$ also increases $\left(\frac{\partial u_{T T}}{\partial t}(x, t)>0\right)$. The aim that we introduce Brownian motion into image denoising is to remove the ill-effects caused by the two cases.

In fact, the Brownian motion used in this proposed method is 1-dimension "random walking" in the gray axis of an image. Its action is to weaken the blurring in $N$-direction and noise amplification in $T$-direction. So the direction of Brownian motion displacement is opposite to diffusion process and its distance is decided by a normal distribution random number whose standard derivation is computed by Eq.(7). For a pixel where $\frac{\partial u_{N N}}{\partial t}(x, t)<0$ or $\frac{\partial u_{T T}}{\partial t}(x, t)>0$, we set a reflection wall at the current diffusion location at time $t$. If $\mathrm{M}_{r}(x, t)$ denotes the location of this reflection wall corresponding to $u(x, t)$, then $\mathrm{M}_{r}=u(x, t)$. We can find that if the variation of gray value of a pixel has the same Brownian motion direction with the diffusion process, then reflection wall will force it move toward opposite direction with the same motion distance. Therefore, the variation of gray value can only occur so as to decrease the ill-effects caused by nonlinear diffusion because of the action of reflection wall.

On the other hand, it is necessary to set the absorption walls for Brownian motion such that Brownian motion does not 
change the convergence of nonlinear diffusion process in the iterations process. We use $\mathrm{M}_{a}(x, t)$ to denote the locations of the absorption walls corresponding to $u(x, t)$. When Brownian motion of a pixel touches to the absorption walls, the motion will be absorbed by the walls rather than perforate through the walls. In other word, the absorption walls limit the range of variation of gray value caused by Brownian motion. According to the “Minimun-Maximun” principle, no new local minimum and maximum should be created at any time. Assuming that $\nabla g_{B}(x, t)$ is the displacement caused by Brownian motion or reflection wall for pixel $x$ at time $t$, then $\nabla g_{B}(x, t)$ should satisfy the flowing equation because of the limitation of the absorption walls $\mathrm{M}_{a}(x, t)$ :

$\min \{(x, 0) m x \in \mathrm{N} \subset \Omega\} \leq \nabla g_{B}(x, t)+u(x, t) \leq \max \{(x, 0) m x \in \mathrm{N} \subset \Omega\}$

where $\mathrm{N}$ is a neighborhood around $x$. There are two absorption walls for a pixel, denoted by $\mathrm{M}_{a}^{\uparrow}$ and $\mathrm{M}_{a}^{\downarrow}$, respectively:

$$
\left\{\begin{array}{l}
\mathrm{M}_{a}^{\uparrow}(x, t)=\max \{u(x, 0), x \in \mathrm{N} \subset \Omega\}-u(x, t) \\
\mathrm{M}_{a}^{\downarrow}(x, t)=u(x, t)-\min \{u(x, 0), x \in \mathrm{N} \subset \Omega\}
\end{array}\right.
$$

Then the absorption walls $\mathrm{M}_{a}(x, t)$ are defined as a vector: $\mathrm{M}_{a}(x, t)=\left[\mathrm{M}_{a}^{\uparrow}(x, t), \mathrm{M}_{a}^{\downarrow}(x, t)\right]^{T}$.

For a pixel where $\frac{\partial u_{N N}}{\partial t}(x, t) \geq 0$ and $\frac{\partial u_{T T}}{\partial t}(x, t) \leq 0$, i.e. nonlinear diffusion has removed noise while preserved edges with well-effects, we forbid the Brownian motion by forcing the absorption walls having the same location with the reflection wall:

$\mathrm{M}_{a}^{\uparrow}(x, t)=\mathrm{M}_{a}^{\downarrow}(x, t)=\mathrm{M}_{r}(x, t)=u(x, t)$.

To summarize, the locations of reflection wall and absorption walls for pixel $x$ at the diffusion time $t$, are written as follows:

$$
\left\{\begin{aligned}
\mathrm{M}_{r}(x, t)=u(x, t) \quad \text { for } \forall x \in \Omega \\
\mathrm{M}_{a}(x, t)=\left(\begin{array}{l}
\mathrm{M}_{a}^{\uparrow} \\
\mathrm{M}_{a}^{\downarrow}
\end{array}\right)=\left(\begin{array}{l}
\max \{l(x, 0), x \in \mathrm{N} \subset \Omega\}-u(x, 0) \\
u(x, 0)-\min \{l(x, 0), x \in \mathrm{N} \subset \Omega\}
\end{array}\right) \\
\text { for } \frac{\partial u_{T T}(x, t)}{\partial t}>0 \text { or } \frac{\partial u_{N N}(x, t)}{\partial t}<0 \\
\mathrm{M}_{a}(x, t)=\left(\begin{array}{l}
\mathrm{M}_{a}^{\uparrow} \\
\mathrm{M}_{a}^{\downarrow}
\end{array}\right)=\left(\begin{array}{l}
u(x, 0) \\
u(x, 0)
\end{array}\right) \text { for } \frac{\partial u_{T T}(x, t)}{\partial t} \leq 0 \text { and } \frac{\partial u_{N N}(x, t)}{\partial t} \geq 0
\end{aligned}\right.
$$

\subsection{The variations of gray values}

In the above subsection, we set the reflection and absorption walls for each pixel in image, and the walls vary their locations according to the local feature of the neighborhood around this pixel. The walls limit the range of variation of gray value of this pixel, in order to keep the stability of inverse process

For a pixel $x$ at current diffusion time $t$,let $\mathrm{M}_{a}^{t}(x, t)=\max \left(\mathrm{M}_{a}^{\uparrow}(x, t), \mathrm{M}_{a}^{\downarrow}(x, t)\right)$ and $\mathrm{M}_{a}^{b}(x, t)=\min \left(\mathrm{M}_{a}^{\uparrow}(x, t), \mathrm{M}_{a}^{\downarrow}(x, t)\right)$ denote the top and bottom absorption wall, respectively.Let $d=\operatorname{sign}(u(x, t)-u(x, t-1))$ denote the direction of nonlinear diffusion process. $r \sim N\left(0, \sigma^{2}\right)$ is the random distance of Brownian motion displacement. With the reflection and absorption walls for this pixel, the variation of gray value of this pixel is written as:

$$
\nabla g_{B}(x, t)= \begin{cases}-\operatorname{sign}(d) \times|r| & \text { if }|r| \in\left(\mathrm{M}_{a}^{b}(x, t), \mathrm{M}_{a}^{t}(x, t)\right) \\ -\operatorname{sign}(d) \times \mathrm{M}_{a}^{b}(x, t) & \text { if }|r| \leq \mathrm{M}_{a}^{b}(x, t) \\ -\operatorname{sign}(d) \times \mathrm{M}_{a}^{t}(x, t) & \text { if }|r| \geq \mathrm{M}_{a}^{t}(x, t)\end{cases}
$$

Form Eq.(11) and (12), we can find that the variation of gray value, $\nabla g_{B}(x, t)$, is always opposite to the nonlinear diffusion process at those location where nonlinear diffusion has ill-effects $\left(\frac{\partial u_{N N}}{\partial t}(x, t)<0\right.$ or $\left.\frac{\partial u_{T T}}{\partial t}(x, t)>0\right)$, and at those location where nonlinear diffusion has well-effects 


$$
\left(\frac{\partial u_{N N}}{\partial t}(x, t) \geq 0 \quad \text { or } \quad \frac{\partial u_{T T}}{\partial t}(x, t) \leq 0 \quad\right) \text {, }
$$

$\nabla g_{B}(x, t)=0$, since the reflection wall is at the same location with the absorption walls. We think that it is Brownian motion that causes the variation of gray value. Its immediate result is to prevent smoothing the edges in $N$-direction and amplifying noise in $T$-direction. So Brownian motion removes the ill-effects of nonlinear diffusion process described in the above subsection.

\subsection{The combination of nonlinear diffusion and Brownian motion}

As an inverse process of nonlinear diffusion, the variation of gray value caused by Brownian motion is introduced into image denoising. Then the iteration expression of combination of nonlinear diffusion and Brownian motion is written as follows, under Neumann boundary conditions:

$u(x, t)=u(x, t-1)+\nabla t \times \operatorname{div}\left(c\left(|\nabla u|^{2} \nabla u\right)+\nabla g_{B}(x, t)\right.$

where $\nabla g_{B}(x, t)$ is computed by Eq.(12). This proposed method is call ND-BM method. Because of the action of reflection and absorption walls, $\nabla g_{B}(x, t)$ is also a bounded function. So $\nabla g_{B}(x, t)$ does not change the convergence of nonlinear diffusion process. The convergence problem of nonlinear diffusion has been discussed deeply in many literatures. We may predict that the proposed method for image denoising needs more iteration than the one where only nonlinear diffusion is used, because the Brownian motion confuse the diffusion process such that the edges are preserved. In our work, we use $\left\|u-u_{0}\right\|^{2}=N^{2} \sigma_{n}^{2}$ as the iteration stopping criteria that was suggested in [6], where $N^{2}$ is the size of image and $\sigma_{n}^{2}$ is the variance of noise signal.

\section{Experimental Results}

We applied this proposed ND-BM method to noisy Lena image to show its capability of image denoising. For comparison purpose, both P-M and FAB method were implemented. Let $u_{0}$ denote the observed image, $u$ denote the original image and $\hat{u}$ denote the approximation of $u$ by any one of the three image denoising methods. The SNR of observed image is $20 \mathrm{db}$. As an objective measurement of an algorithm performance, the improvement in signal-to-noise-ratio(ISNR)is defined as:

$$
I S N R=10 \times \log _{10}\left(\frac{\left\|u-u_{0}\right\|^{2}}{\|u-\hat{u}\|^{2}}\right)
$$

We also use Local Variance Mean Square Error( $L V M S E)$ as the other measurement of an algorithm performance. LVMSE has been discussed in [7]. It is computed by:

$$
\text { LVMSE }=\frac{1}{N^{2}} \sum_{x \in \Omega}\left[\sigma_{L}^{2}(u(x))-\sigma_{L}^{2}(\hat{u}(x))\right]^{2}
$$

where $\sigma_{L}^{2}(u(x))$ and $\sigma_{L}^{2}(\hat{u}(x))$ are the variance of the local region surrounding pixel $u(x)$ in original image and $\hat{u}(x)$ in the denoisy image.

The experimental data is listed in Table.1. Form Table.1, we can find that the denoisy image that are obtained by this proposed NF-BM method have the better evaluation in both ISNR and LVMSE than by $\mathrm{P}-\mathrm{M}$ method and FAB method.

Table 1: the ISNR and LVMSE of the different methods

\begin{tabular}{c|c|c|c}
\hline & P-M & FAB & ND-BM \\
\hline$I S N R(\mathrm{~dB})$ & 0.328 & 1.119 & 2.830 \\
\hline $\operatorname{LVMSE}\left(\times 10^{5}\right)$ & 25.922 & 3.634 & 3.630 \\
\hline
\end{tabular}


Fig.2(c) shows that the denoisy image obtained by P-M method is over-smoothness, some detail and edges are blurred, although noise is removed sufficiently. From Fig.2(d) and (e) show the denoisy images obtained by the FAB and the proposed ND-BM methods respectively. We can find that the both methods preserve the edges successfully. But the proposed method removes more noise in smoothing region. This phenomenon is revealed more obviously in the corresponding local magnified image.

The iteration processes of the three methods are stopped when $\left\|u_{0}-\hat{u}(t)\right\|^{2}$ is first larger than $N^{2} \sigma_{n}^{2}$. Their convergence curves are shown in Fig.3. We can find that the convergence of $\mathrm{P}-\mathrm{M}$ method is almost linear, and FAB has faster speed in the finial stage. There is some slight oscillation in ND-BM convergence curve, because of the effect of "random walking" of Brownian motion. It also shows that the ND-BM method needs a little more time to obtain higher quality denoisy image.
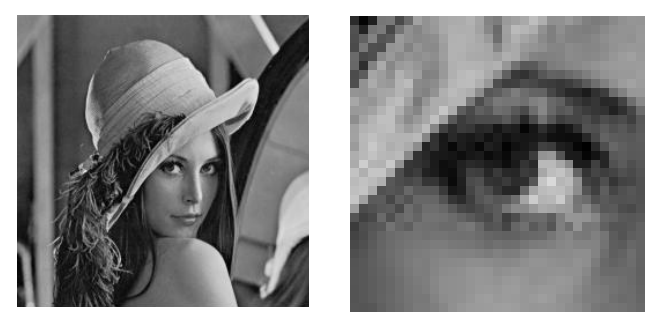

(a)
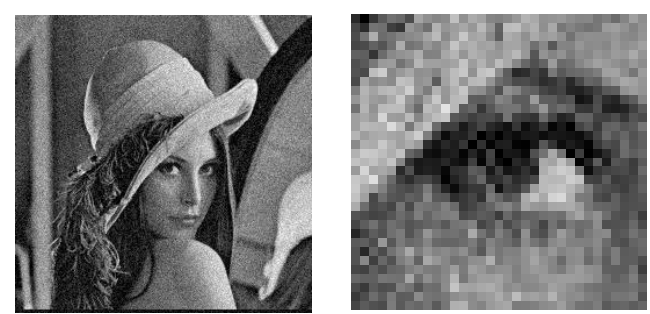

(b)
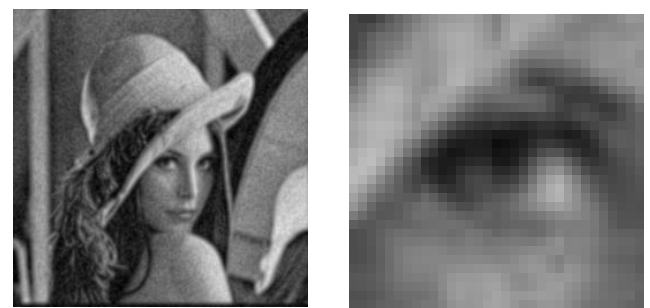

(c)
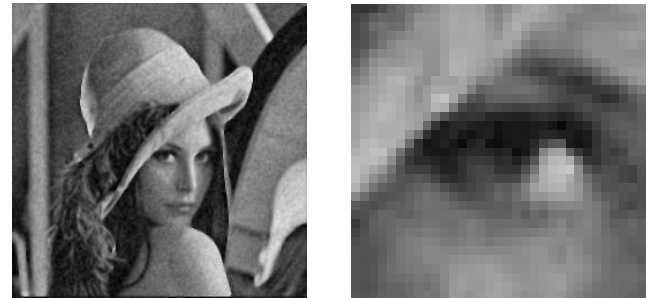

(d)
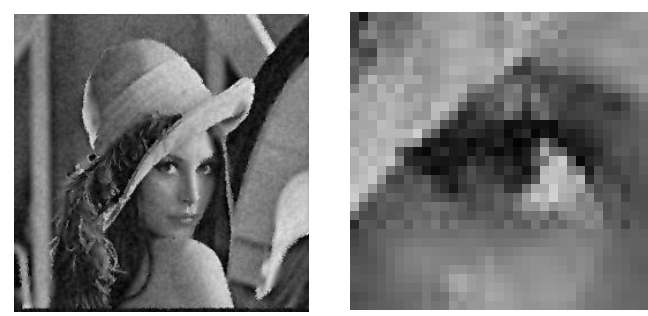

(e)

Fig2. the experimental results images: (a) the original image; (b) the noisy image(observed image); (c) the denoisy image by P-M method; (d) the denoisy image by FAB method and (e) the denoisy image by the proposed ND-BM method.

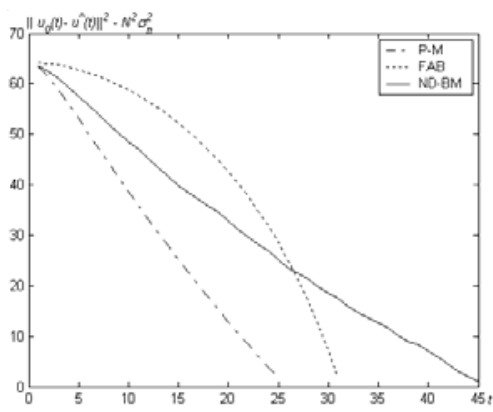

Fig3. the convergence curves of differen 


\section{Conclusion}

Brownian motion of a pixel analogs the irregular and unceasing movement of a small particle. In image denoising, because of the effect of reflection wall, Brownian motion acts as the inverse process of nonlinear diffusion, such that it prevents that the edge is blurred in the orthogonal direction of the edge and noise is magnified in its tangent direction. The absorption walls limit the range of variation of gray value of each pixel, therefore, the stability of the solution is guaranteed. The experimental results show that the proposed method of the combination of nonlinear diffusion and Brownian motion removes noise while preserves the edge more effectively.

\section{Acknowledgmentz}

This work is financially supported by National Natural Science Foundation of China(60702032), and Natural Scientific Research Innovation Foundation in Harbin Institute of Technology(HIT.NSRIF.2008.63).

\section{References}

[1] M.Nielsen, L.Florack and R.Deriche. "Regularization, scale-space and edge detection filters". Journal of Mathematical Imaging and Vision, pp. 291-307. 1997 July.

[2] P.Peroma and J.Malik. "Scale-space and edge detection using anisotripic diffusion". IEEE Transactions on Pattern Analysis and Machine Intelligence, pp. 629-639, 12(7),1990.

[3] F.Catte,L.Lions and .Morel. "Image selective smoothing and edge detection by nonlinear diffusion". SIMA J.Num.Anal., pp. 182-193, 29(1),1992.
[4] G.Giboa,N.Sochen, Y.Zeevi."Forward-and-backward diffusion for adaptive image enhancement and denoising”, IEEE Transactions on Image Processing, pp. 689-703, 11(6),2002.

[5] G.Aubert and P.Kornprobst. "Mathematical Problems in Image Processing: Partial Differential Equations and The Calculus of Variations”. Springwe-Verlag, 2000.

[6] L.Rudin,S.Osher and E.Fatemi. "Nonlinear total variation based noise removal algorithm". Physica D, pp. 259-268, 60,1992.

[7] A.S.Palmer, H.S.Wong and L.Guan. “ Adaptive image processing: A computational intelligence perspective. CRC Press. New York,USA,2002.

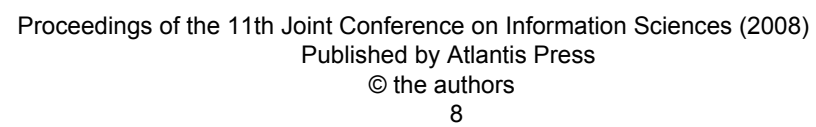

